\title{
Predicting HLA genotypes using unphased and flanking single-nucleotide polymorphisms in Han Chinese population
}

\author{
Ai-Ru Hsieh ${ }^{1,13+}$, Su-Wei Chang ${ }^{2 \dagger}$, Pei-Lung Chen ${ }^{3,4,11,12}$, Chen-Chung Chu ${ }^{10}$, Ching-Lin Hsiao ${ }^{1}$, Wei-Shiung Yang ${ }^{3,4,5,11}$, \\ Chien-Ching Chang ${ }^{1}$, Jer-Yuarn Wu ${ }^{1,7,8}$, Yuan-Tsong Chen ${ }^{1,7,9}$, Tien-Chun Chang ${ }^{4,6^{*}}$ and Cathy SJ Fann ${ }^{*^{*}}$
}

\begin{abstract}
Background: Genetic variation associated with human leukocyte antigen (HLA) genes has immunological functions and is associated with autoimmune diseases. To date, large-scale studies involving classical HLA genes have been limited by time-consuming and expensive HLA-typing technologies. To reduce these costs, single-nucleotide polymorphisms (SNPS) have been used to predict HLA-allele types. Although HLA allelic distributions differ among populations, most prediction model of HLA genes are based on Caucasian samples, with few reported studies involving non-Caucasians.
\end{abstract}

Results: Our sample consisted of 437 Han Chinese with Affymetrix 5.0 and Illumina 550 K SNPs, of whom 214 also had data on Affymetrix 6.0 SNPs. All individuals had HLA typings at a 4-digit resolution. Using these data, we have built prediction model of HLA genes that are specific for a Han Chinese population. To optimize our prediction model of HLA genes, we analyzed a number of critical parameters, including flanking-region size, genotyping platform, and imputation. Predictive accuracies generally increased both with sample size and SNP density.

Conclusions: SNP data from the HapMap Project are about five times more dense than commercially available genotype chip data. Using chips to genotype our samples, however, only reduced the accuracy of our HLA predictions by only 3\%, while saving a great deal of time and expense. We demonstrated that classical HLA alleles can be predicted from SNP genotype data with a high level of accuracy (80.37\% (HLA-B) 95.79\% (HLA-DQB1)) in a Han Chinese population. This finding offers new opportunities for researchers in obtaining HLA genotypes via prediction using their already existing chip datasets. Since the genetic variation structure (e.g. SNP, HLA, Linkage disequilibrium) is different between Han Chinese and Caucasians, and has strong impact in building prediction models for HLA genes, our findings emphasize the importance of building ethnic-specific models when analyzing human populations.

Keywords: Major histocompatibility complex (MHC), Human leukocyte antigen (HLA), Single-nucleotide polymorphisms (SNPs)

\section{Background}

With the advent of high-throughput genotyping technologies, it is now relatively easy to obtain large-scale, genomewide data concerning single-nucleotide polymorphisms (SNPs) in humans. This allows for more thorough analyses of questions that involve population genetics. Multiple

\footnotetext{
* Correspondence: tienchunchang@ntu.edu.tw; csjfann@ibms.sinica.edu.tw ${ }^{\dagger}$ Equal contributors

${ }^{4}$ Department of Internal Medicine, National Taiwan University Hospital, Taipei, Taiwan

${ }^{1}$ Institute of Biomedical Sciences, Academia Sinica, Nankang, Taipei, Taiwan Full list of author information is available at the end of the article
}

SNPs often cover or flank functionally important genes, such as human leukocyte antigen (HLA) genes that comprise the major histocompatibility complex (MHC) in humans. The $H L A$ s localize to chromosome $6 \mathrm{p}$ and include MHC class I (HLA-A, HLA-B, and HLA-C) and MHC class II (HLA-DR, HLA-DQ and HLA-DP) genes. Unlike bi-allelic SNPs, $H L A$ genes are extremely polymorphic. Currently, more than 8794 alleles for the $H L A$ loci have been identified in various populations, including 2862 HLA-B alleles according to the IMGT/HLA database 3.11.0 version [1]. Mismatched HLA alleles can lead to

\section{Biomed Central}


graft rejection and graft-versus-host diseases [1,2]. HLA genes also play critical roles in both population genetics and immune-related disease status $[3,4]$. Furthermore, previous comparative studies have shown that immune systems are generally under strong selective pressures, which are likely driven by virus-host interactions $[5,6]$. Because of these selective pressures, comparisons between ethnic groups reveal linkage disequilibrium and highly variable patterns of allelic distributions for $H L A$ genes [5,7].

The Taiwanese comprised the Minnan and Hakka people groups. The genetic profile of the Taiwanese shows many affinities to southern Asian populations [8]. HLA-B* $4601 / B 46$ displays higher frequencies in Southern Han (15.4\%), Singaporean (15.1\%) and Vietnamese (13.2\%) than in Northern Han (2.8\%) [9]. There were a few studies that have focused on the association between HLA markers and disease using Southern Han Chinese data. Most of the studies found them a homogeneous group [8-11].

Recent advances in array-based SNP genotyping technologies have led to a revolution in the nature and scale of disease-association studies. These developed technologies yield economical ways to genotype one million SNPs across the entire genome in large-population studies. To date, direct experimental methods for typing $H L A$ genes (via serology or PCR) remain laborious, expensive, and time consuming. This has limited large-scale studies involving HLAs $[5,12]$. To reduce the cost of these studies, researchers have begun to use data from SNPs around the $H L A$ regions to make predictions concerning $H L A$ allele types [13,14]. Leslie et al. [14] developed a statistical method based on identity by descent, which uses phaseresolved genotype data (i.e. full haplotype information was inferred by multiple SNPs) to predict $H L A$ alleles. Li et al. [13] proposed a complementary method for predicting $H L A$ alleles based on unphased genotype data. Given a training data set of unphased genotypes and their corresponding phased $H L A$ alleles, they used a likelihood method to compute probabilities associated with all possible pairs of $H L A$ alleles and their particular haplotypes. Because of high-throughput SNP-genotyping technologies, the method developed by Li et al. [13] analyzes many lesscommon HLA alleles. This results in more accurate predictions concerning $H L A$ alleles.

After a decade of research, $H L A$ prediction methodologies have achieved a high level of accuracy. For classical $H L A$ class I and class II genes, there is typically a 97\% accuracy when Caucasian populations are analyzed $[13,14]$. These types of studies, however, have not been rigorously applied to other ethnic groups [7]. Zhang et al. [7] revealed that predictive accuracies were poor if $H L A$ alleles from non-Caucasian subjects were used as the training data set to build predictive $H L A$ models for different ethnic groups. Hence, the African-specific $H L A$-prediction model has been developed [15]. Unfortunately, there is very little information concerning $H L A$ allele prediction in Han Chinese populations. This need must be addressed before effective, ethnic-specific prediction model of $H L A$ genes can be formulated.

Leslie et al. [14] achieved a high level of accuracy using data from the Haplotype Map (HapMap) Project as the training data. They validated their $H L A$-prediction model using genotypes and $H L A$ allele information from the British 1958 birth cohort study. Unfortunately, genotype datasets with densities that approach the HapMap Project are not commonly available. As such, the use of chip data may represent an alternative method for constructing prediction model of $H L A$ genes that are specific for Han Chinese populations.

Toward this goal, we applied the Li et al. [13] method to samples from Taiwan to build ethnic-specific prediction model of HLA genes for Han Chinese populations. This study comprised 437 Han Chinese with Affymetrix 5.0 and Illumina $550 \mathrm{~K}$ SNPs, of whom 214 also had data on Affymetrix 6.0 SNPs. All individuals were $H L A$ typed at a $4-$ digit level resolution at $6 \mathrm{HLA}$ loci and were used for training and testing the prediction model of $H L A$ genes. To optimize these prediction models for classical HLA class I and class II genes, we addressed the following questions: 1) would there be differences in $H L A$ allele distributions and optimal flanking regions between our Han Chinese data set and the HapMap Caucasian samples, 2) could MHC SNP data generated by different platforms yield comparably accurate $H L A$ allele predictions, and 3) could imputation of untyped MHC SNPs improve the accuracy and robustness of the model? We provide practical recommendations concerning ethnic-specific prediction model of $H L A$ genes regarding $H L A$ gene regions, platforms, and imputation.

\section{Methods}

\section{Ethics statement}

Blood samples from 437 Han Chinese subjects residing in Taiwan were obtained from the Taiwan Han Chinese Cell and Genome Bank [16] and were used for this analysis. This study was approved by the Internal Review Board of Academia Sinica. A written informed consent was signed by every participant at his/her initial clinic visit. All individuals in this study were Han Chinese. All participants in this study have full Han ethnicity through both maternal and paternal grandparents and familial residence in the area of Taiwan for the last 3 generations. Our data consisted of three Taiwanese subgroups: Minnan (70\%), Hakka (13\%) and Mainlanders (14\%). In one of our previous study for MHC SNPs in Han Chinese residing in Taiwan, we have shown that the Taiwanese population is homogeneous [17]. 


\section{DNA samples and MHC SNP data}

Genomic DNA was extracted from blood using the Puregene DNA Isolation Kit (Gentra Systems, Minneapolis, USA). Four hundred and thirty seven samples were genotyped using the Affy 5.0 (Affymetrix, Santa Clara, CA, USA) and the Illumina $550 \mathrm{~K}$ chips (Illumina, San Diego, CA, USA). Two hundred fourteen of these samples were also genotyped using the Affy 6.0 chip. The Affy 5.0 chip contains 31,393 SNPs on chromosome 6 among 488,756 SNPs across 22 chromosomes. The Affy 6.0 chip contains 56,202 SNPs on chromosome 6 among 906,600 SNPs across 22 chromosomes. The Illumina $550 \mathrm{~K}$ chip contains 36,591 SNPs on chromosome 6 among 546,401 SNPs across 22 chromosomes. The National Center for Genome Medicine at Academia Sinica carried out all genotyping. All of the sample call rates were $>97 \%$, and the mean individual-sample call rate was $98.4 \pm 0.7 \%$.

Within the extended human MHC region, which includes $\sim 6$ megabases $(\mathrm{Mb})$ on chromosome $6 \mathrm{p}$ (position 28-34 Mb), the Affy 6.0 chip contains 2,203 SNPs, the Affy 5.0 chip contains 1,406 SNPs, and the Illumina $550 \mathrm{~K}$ chip contains 1,939 SNPs (Additional file 1). The intra-MHC region spanning the $H L A-A$ gene at the telomeric end and the $H L A-D P B 1$ gene at the centromeric end actually harbors class III genes (which are also termed complement genes), in addition to both class I loci ( $H L A-A$, $H L A-B, H L A-C)$ and class II loci (HLA-DRB1, HLA-DQB1, HLA-DPB1).

\section{HLA genotyping}

$H L A$ genotypes were observed from our previous study [18]. Briefly, six classic $H L A$ genes (HLA-A, $-B,-C$, $-D Q B 1,-D R B 1$, and $-D P B 1)$ to a 4-digit resolution were analyzed in the present study. Among these genes, the $H L A-A,-B,-C,-D Q B 1$, and $-D R B 1$ alleles were genotyped using the Dynal RELI SSO typing kit (Dynal Biotech Ltd., Bromborough, Wirral, UK; now part of Life Platforms, Carlsbad, CA, USA). In brief, both exon 2 and exon 3 of class I genes (HLA-A, $-B$ and $-C)$ and exon 2 of class II genes (HLA-DQB1 and $-D R B 1)$ were amplified by PCR using locus-specific primer sets. After amplification, PCR products were hybridized to sequence-specific oligonucleotide (SSO) probes that had been previously fixed to a nylon membrane in a linear array. SSO probes included $48 H L A-A$ probes, $61 H L A-B$ probes, $37 H L A-C$ probes, 41 HLA-DQB1 probes, and $60 H L A-D R B 1$ probes. The Pattern-Matching Program (Dynal Biotech, Ltd.) was used to interpret genotypes. Because the Dynal RELI SSO system lacked an HLA-DPB1 genotyping kit, HLA-DPB1 was genotyped using the Gold SSP HLA-DPB1 High Resolution Kit (Invitrogen; now part of Life Platforms; California, USA). This genotyping technique is based on the sequencespecific primer amplification method. The HLA-DPB1 genotyping was based on 48 PCR reactions for each DNA sample. The UniMatch software (Invitrogen) used the pattern of PCR amplification to interpret HLA-DPB1 genotypes. While medium to high resolution typing results were obtained from those reverse SSO typing systems, several genotype combinations produced same reaction patterns. For these conditions, allele designation were assigned according to the most common alleles (allele frequency $>0.01$ ) found in Taiwanese populations and southern Chinese populations as determined in the population studies of $13^{\text {th }}$ international histocompatibility workshop or to the allele with the lowest definition number. The four digit alleles detected at each $H L A$ locus and allele frequencies in our Han Chinese data $(\mathrm{n}=214)$ were listed in Additional file 2.

\section{HLA prediction methodology}

To build prediction model of HLA genes using unphased genotypes, we adopted an estimating equation approach [13]. For each gene, the $H L A$-predictive methodology was then carried out as two separate procedures. The first procedure constructed a prediction model, whereas the second procedure validated the model generated by the first procedure. In the first procedure, a set of unphased genotypes was selected to build a prediction model. This selection process was evaluated using an objective function [13], which was the negative log-likelihood of the $H L A$ allele given unphased genotypes (based on the Akaike Information Criterion) [19]. Genotype selection was then performed using the forward-selection and backwardelimination scheme. This started with genotypes associated with an $H L A$ allele and we gradually added one genotype at a time. The second procedure validated the prediction model using an independent set of samples. For these independent samples, unphased genotypes and phased $H L A$ alleles were provided. Following the parsimonious rule, the best prediction model should use the smallest possible flanking region and the fewest possible SNPs to generate the most accurate predictions.

\section{Scenarios \\ Differences in HLA allele frequency distributions and flanking-region sizes between ethnic groups}

$H L A$ alleles and their allelic distributions differ substantially between ethnic groups, reflecting their recent evolutionary histories $[7,20]$. Furthermore, $H L A$ genes cover different regions on chromosome $6 \mathrm{p}$ and include various numbers of SNPs [12]. Here we explored allele frequency distributions within our Han Chinese samples and within Caucasian samples from the HapMap Project [21]. For each $H L A$ allele, we used chi-square and Fisher's exact tests to determine whether $H L A$ allele proportions were different between these two populations. Following Li et al.'s [13], we assessed flanking regions that extended $\pm 10 \mathrm{~kb}$ to $\pm 400 \mathrm{~kb}$ to construct the prediction model of HLA genes. Within the Han Chinese population, the most appropriate flanking 
region for each $H L A$ gene was determined using the parsimonious rule described above. Furthermore, we compared flanking-region sizes derived from our Han Chinese population (from the Affy 5.0 chip) with flanking-region sizes from the Caucasian population in Li et al.'s [13].

\section{Different platforms}

Three platforms were used in this study: 1) the Affymetrix Genome-Wide Human SNP Array 5.0 (Affy 5.0) [22,23], 2) the Affymetrix Genome-Wide Human SNP Array 6.0 (Affy 6.0), and 3) Illumina's HumanHap550 Genotyping BeadChip (Illumina 550) [24,25]. To measure the compatibility between these platforms we compared overlapping SNP data from each pair of platforms. Genotype data concordance was computed using Cohen's kappa coefficient [26], a measurement commonly used to assess the degree of concordance between two independent groups [7]. Values of kappa $>0.9$ generally indicate excellent reliability [27]. For each platform pair, genotype concordance was evaluated between all observed SNPs and all imputed SNPs. For each pair, we also compared variation between genotypes that were selected during the HLA modelbuilding process. This was done to determine whether selected genotypes were platform specific. Variation was defined as $\frac{U\left(\text { plat }_{i}, \text { plat }_{j}\right)-\cap\left(\text { plat }_{i}, \text { plat }_{j}\right)}{U\left(\text { plat }_{i}, \text { plat }_{j}\right)}$, where plat $_{i}$ and plat $_{j}$ are two different platforms, $\cup\left(\right.$ plat $_{i}$, plat $\left._{j}\right)$ is the union of the SNPs for two different platforms, and $\cap\left(\right.$ plat $_{i}$, plat $\left._{j}\right)$ is the intersection of the SNPs for two different platforms.

\section{Imputation}

"Genotype imputation" is the term used to describe the process of imputing genotypes that are not directly assayed in a sample of individuals [28-30]. Genotype imputation has become a routine practice in genome-wide association studies (GWASs). Here we evaluated the usefulness of imputed genotypes in constructing prediction model of $H L A$ genes. For data consistency and optimal-imputation performance, we used the $\mathrm{MaCH}$ [31] software and the Chinese Han Beijing (CHB) + Japanese Tokyo (JPT) data set [32] as a reference. This was used to impute genotypes beyond our SNPs, which were derived from the HapMap Project. Standard GWAS quality-control filters (e.g., minorallele frequency $<0.01$, genotype call rates $<0.95$, and a significant departure from Hardy-Weinberg equilibrium at $\mathrm{p}<10^{-4}$ ) are usually sufficient before genotype imputation [33-35]. We applied these recommendations, therefore, and checked our genotype data for all SNPs within the MHC region. Furthermore, each imputed SNP that were included in this study should have an imputation posterior probability from $\mathrm{MaCH}>0.8$, call rate $>0.95$, and minor-allele frequency $>0.01$. The three steps used for genotype imputation were as follows: 1) apply standard GWAS quality-control filters to our MHC SNPs, 2) use $\mathrm{MaCH}$ software to impute genotypes beyond MHC SNPs that were determined in step 1, and 3) check the imputation posterior probabilities from $\mathrm{MaCH}$ for the imputed SNPs that were determined in step 2.

\section{Cross-validation}

Before beginning the $H L A$ prediction analysis, we divided the data into multiple partitions for cross-validation $(\mathrm{CV})$. All 437 individuals were $H L A$ typed at a 4-digit level resolution at $6 H L A$ loci and were used for training and testing the prediction model of $H L A$ genes. We used a 10 -fold $\mathrm{CV}$ in the study, the data set was divided into a training data set (9/10 of the 437 data) and a testing data set $(1 / 10$ of the 437 data). The testing accuracy was assessed according to the comparison between the original $H L A$ alleles and predictive $H L A$ alleles for each sample. If the pair of $H L A$ alleles between the original $H L A$ alleles and predictive $H L A$ alleles was the same, the predictive value was assigned 1. If a half pair of $H L A$ alleles was the same, then the predictive value was assigned 0.5 , else it was assigned 0. For each CV subset, the testing accuracy was calculated for the testing set and defined as $\frac{T_{v}}{N_{v}}$, where $T_{v}$ is the number of correctly predicted samples (the sum of the predictive values from each predicted sample) in the testing set and $N_{v}$ is the total number of samples in the testing set. The average testing accuracy is the mean of the $10 \mathrm{CV}$ subsets and indicates how well the constructed $H L A$ model predicts the HLA alleles. The HLA prediction can be performed without $\mathrm{CV}$, but $\mathrm{CV}$ execution can avoid over-fitting the prediction model and can save both time and costs associated with attaining an independent set of samples for validation [36]. We built prediction model of HLA genes, therefore, using 10-fold CV.

\section{Confidence threshold (CT)}

For each sample within the testing data set, probability values were assigned to each possible pair of $H L A$ alleles given a particular haplotype. These values were based on the provided unphased genotypes and phased pairs of $H L A$ alleles. After assigning probabilities, we selected the pair with the maximum probability, if that probability exceeded a pre-specified CT. When CT is set to be 0 , it means that the call rate is $100 \%$ (i.e., all samples will be predicted). If CT is set to be 0.5 (or any value greater than 0 ), only samples for which the maximum predictive probability exceeds CT will be used. Here we set CT to be $0,0.5$, or 0.9 to evaluate the effects of $\mathrm{CT}$ on constructing prediction model of $H L A$ genes. If a higher CT is applied, the prediction model of HLA gene results in greater prediction accuracy. 


\section{Results}

$H L A$ allele frequency distributions and flanking-region sizes We calculated allele frequency distributions for the six classical HLA genes using 214 samples that were genotyped using three different genomic platforms (Affy 5.0, Affy 6.0, and Illumina $550 \mathrm{~K}$ chips). Analyzed samples were from Han Chinese subjects living in Taiwan provided by the Han Chinese Cell and Genome Bank [16]. We also analyzed 180 Caucasian samples from the HapMap Project, although $H L A-D P B 1$ data were not available for these samples. The Caucasian samples from HapMap were CEPH samples and only founders were included. The most polymorphic $H L A$ locus was $H L A-B$. Across the $H L A-B$ region, we observed 44 alleles in our Han Chinese population and 32 alleles in the HapMap Caucasians. To address the issue of a sparse contingency table, the allele frequency for $H L A-A,-B,-C,-D Q B 1$, and $-D R B 1$ genes between Caucasians and Han Chinese was compared using program clump (http://www.smd. qmul.ac.uk/statgen/dcurtis/software.html) [37]. All distribution differed significantly between the Caucasians and Han Chinese at the adjusted 5\% level using Bonferroni's correction. We confirmed the previous finding, therefore, that $H L A$ allele frequency distributions differ extensively across populations. As such, predictive $H L A$ models that are built using $H L A$ alleles from one population may generate poor predictions concerning a different ethnic population. Ethnic-specific prediction model of $H L A$ genes are generally preferred [7].

Using the $H L A$-predictive methodology described above, we evaluated testing accuracies given various flankingregion sizes following Li et al.'s [13]. We assessed flanking regions that were extended from $\pm 10 \mathrm{~kb}$ to $\pm 400 \mathrm{~kb}$ (the testing accuracies remained almost the same after $\pm 300 \mathrm{~kb}$ across 6 classical HLA genes). Within the Han Chinese population, the most appropriate flanking region for each $H L A$ gene for prediction was determined using the parsimonious rule. For most of the prediction models (HLA-A, $-B,-C,-D Q B 1,-D R B 1$, and -DPB1), trends in the data indicated that more-accurate results were obtained when larger flanking regions were used (Figure 1). The number of predictive SNPs selected also increased with the size of the flanking region. The most dramatic effects on testing accuracy were observed for $H L A$ $D R B 1$ and $H L A-D Q B 1$. When a $\pm 10 \mathrm{~kb}$ flanking region was used, $H L A-D R B 1$ yielded the lowest testing accuracy (68.51\%). This accuracy increased to $>85 \%$ when the flanking region was increased to $\pm 50 \mathrm{~kb}$. For $H L A-D Q B 1$, testing accuracy ranged from $80.75 \%$ ( $\pm 10 \mathrm{~kb}$ flanking region) to $95.79 \%$ ( $\pm 40 \mathrm{~kb}$ region). Once the size of the flanking region increased to $\pm 30 \mathrm{~kb}$, the testing accuracy of $H L A-D Q B 1$ increased to $>90 \%$. Testing accuracies for HLA-C (92.02\%) and HLA-DPB1 $(87.42 \%)$ were satisfactory at $\pm 10 \mathrm{~kb}$. Notably, testing accuracies for $H L A-A(74.52 \%)$ and $H L A-B$
(77.34\%) were particularly low with $\pm 10 \mathrm{~kb}$ flanking regions. Using the size $\pm 150 \mathrm{~kb}$, however, raised the accuracy to $>80 \%$ for both genes. Flanking-region results across the different genotyping platforms (Affy 5.0, Illumina $550 \mathrm{~K}$, and a union of the three platforms) are provided in Additional file 3 .

We also compared flanking-region sizes derived from our Han Chinese population with those from the Caucasian population described previously by Li et al.'s [13]. HLA-A gene required the largest flanking regions $( \pm 200 \mathrm{~kb}$ for the Han Chinese and $\pm 350 \mathrm{~kb}$ for the Caucasian samples, respectively). For $H L A-C, \mathrm{a} \pm 20 \mathrm{~kb}$ region leads to an accuracy of $90.87 \%$ which is the best model by our definition for the Han Chinese, however, it was $\pm 180 \mathrm{~kb}$ for the Caucasian population ( $\mathrm{Li}$ et al. [13]). The size difference in the optimal flanking region was even more dramatic for $H L A-D Q B 1$, which was $\pm 40 \mathrm{~kb}$ for the Han Chinese and $\pm 300 \mathrm{~kb}$ for the Caucasians. The comparisons of flanking region were based on Affy 5.0 chip (Table 1) which was used by Li et al.'s [13] (data not shown). D' values was also listed in Table 1. The flanking region sizes from Caucasian described previously by Li et al.'s [9] were different from ours (Affy 5.0) probably due to population stratification. From our chip data, the range of the highest D' for the $H L A$ markers was between 0.47 and 0.83 without imputation. It was 0.51 to 0.85 with imputation. $H L A-C$ had the highest D' regardless of imputation and platform while HLA-DPB1 had the lowest D'. $H L A-A$ had the longest flanking region regardless imputation or not while HLA-DQB1 had the shortest flanking region. The length of the $H L A$ gene and the LD intensity is negative. However, the LD intensity might not be related to the flanking region size for the optimized predictive models for each platform (Table 1).

\section{Predictive accuracies without imputation}

The overlapping data between each pair of platforms was quite few (Additional file 1). Affy 6.0 had the most SNPs within the MHC regions, whereas Affy 5.0 had the fewest (Additional file 1). Additional file 4 shows kappa coefficients between paired platforms, with respect to observed genotypes. Comparing the two Affymetrix arrays, the kappa coefficient was as high as 0.9926 for the genotypes present on both arrays. This high level of concordance indicated high-quality genotyping, which was further supported by the determination of comparable genotypes between platforms.

In general, Union generated more accurate $H L A$-allele predictions than did each of the individual platforms. For $\mathrm{CT}=0$, the average testing accuracy was $89.78 \%$ with Union, but $86.92 \%, 88.42 \%$, and $88.06 \%$ for Affy 5.0, Affy 6.0 , and Illumina $550 \mathrm{~K}$, respectively (Figure 2A). These findings were consistent with Zhang et al. [7], who 


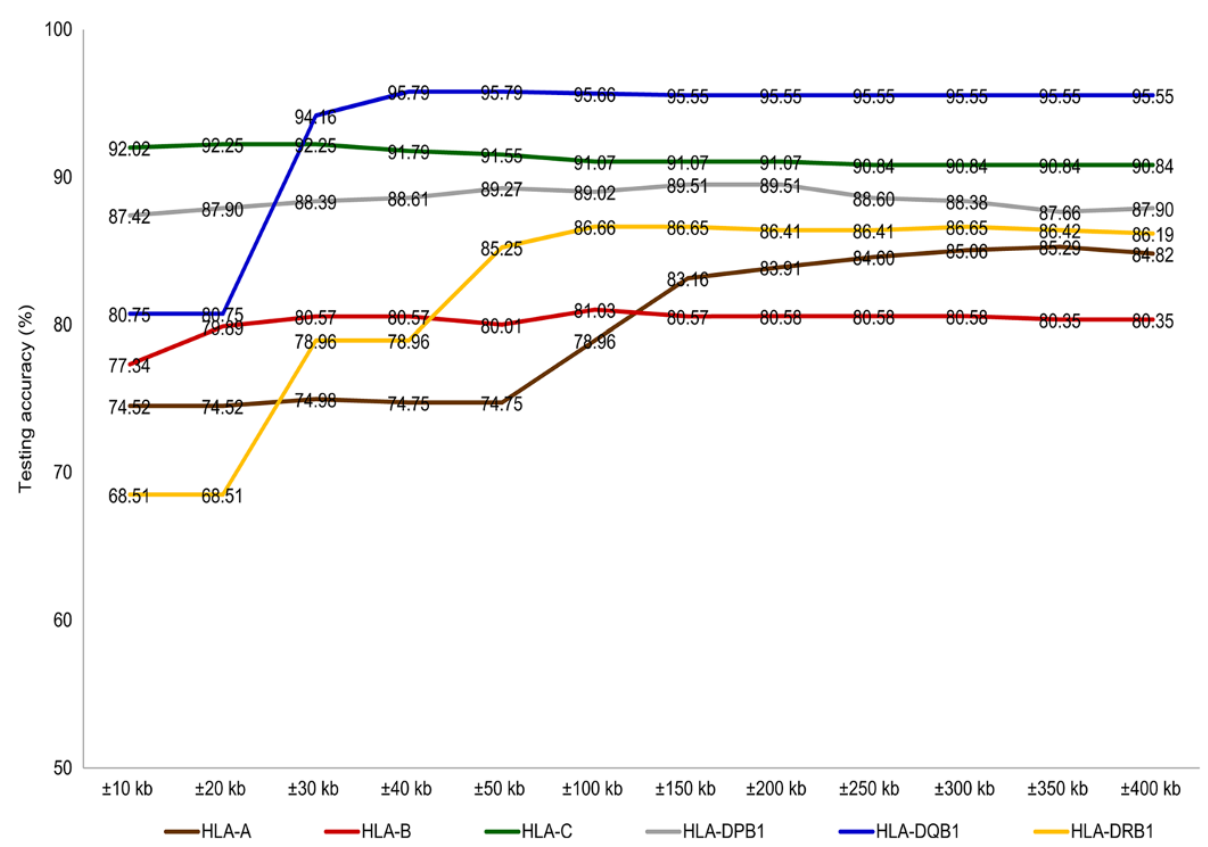

Figure 1 Testing accuracies associated with different flanking-region sizes. For each of the six HLA genes (colored lines), testing accuracies are shown for increasing flanking-region sizes. Data from the Affy 6.0 chip are shown without imputed SNPs.

suggested that higher SNP densities increased the accuracy of $H L A$-allele predictions.

In comparisons between the three genotyping platforms, Affy 6.0 generated the most-accurate $H L A$-allele predictions. For example, Affy 6.0 was $3.52 \%$ more accurate than Affy 5.0 at the HLA-DRB1 locus; and Affy 6.0 was $2.58 \%$ more accurate than Illumina $550 \mathrm{~K}$ at the HLA-DPB1 locus. It was possible that Affy 6.0 did have the highest density of genotypes within the $\mathrm{MHC}$ region. For $\mathrm{CT}=0$, the overall highest testing accuracy was obtained with Affy 6.0 and $H L A-D Q B 1$ (95.79\%), whereas the lowest accuracy was from Illumina $550 \mathrm{~K}$ and $H L A-B$ (80.37\%; Figure $2 \mathrm{~A})$. By applying a CT of 0.9 to the maximum probability of all possible pairs of $H L A$ alleles, the highest accuracy was increased to $98.62 \%$ at the $H L A-C$ locus (with a call rate of $77.47 \%$ from Illumina $550 \mathrm{~K}$ ), whereas the lowest accuracy was elevated to $87.67 \%$ at $H L A-B$ (with a call rate of 64.94\% from Affy 5.0; Additional file 5). The range of accuracies generated by Illumina $550 \mathrm{~K}$-based prediction models was more dramatic across $H L A$ loci than was seen with the other genotyping platforms. With $\mathrm{CT}=0$, the accuracy of the Illumina $550 \mathrm{~K}$ prediction model was only $80.37 \%$ at the $H L A-B$ gene but was $95.29 \%$ at $H L A-D Q B 1$. The Affy 5.0 predictions were $0.45 \%$ and $0.96 \%$ more accurate than Illumina $550 \mathrm{~K}$ for the $H L A-B$ and $H L A-D P B 1$ genes, respectively. Illumina $550 \mathrm{~K}$ predictions were $1.56 \%$ and $0.27 \%$ more accurate than Affy 6.0 for the $H L A-A$ and $H L A-D R B 1$ genes, respectively (Figure 2). These results agreed with Zhang et al.s [7], who showed that slight improvements associated with Affy 5.0 and Illumina $550 \mathrm{~K}$ may be attributed to unique SNPs on these platforms. Overall, accuracies of these prediction models were generally comparable across genotyping platforms. Details concerning testing-accuracy results and the call rate for predictions across different platforms and different $\mathrm{CTs}$ are provided in Additional file 5.

The investigation into the sufficient flanking regions (i.e., the shortest stretch of flanking genomic sequence that yielded the most accurate $H L A$-allele prediction) was performed. The shortest sufficient flanking region was identified at the $H L A-C$ locus using Illumina $550 \mathrm{~K}$ $( \pm 10 \mathrm{~kb})$. The length of $H L A-C$ was $3,325 \mathrm{bp}$ and this sufficient flanking region covered 22 SNPs, of which 13 were incorporated into the $H L A-C$-prediction model (testing accuracy of $92.01 \%$ at $\mathrm{CT}=0$ ). The longest sufficient flanking region was $\pm 350 \mathrm{~kb}$ at $H L A-A$ when Affy 6.0 data were used (Table 1). Within this region, there were 299 SNPs, of which 16 were incorporated into the HLA-Aprediction model (testing accuracy of $85.29 \%$ at $C \mathrm{~T}=0$ ).

For each HLA gene, we further assessed betweenplatform overlap of the genotypes which were incorporated into the $H L A$ prediction model (Additional file 6). The largest percentage of overlapping genotypes for data without imputed SNPs was $21.36 \%$ at HLA-DRB1 when Affy 6.0 and Union were compared. These findings suggest that different platforms might use unique SNPs to select platform-specific genotypes. These genotypes were then used to build the different prediction model of $H L A$ genes. We listed the SNPs that best predict the HLA alleles using the genotype information currently available 
Table 1 Flanking regions associated with optimized prediction models for each platform

\begin{tabular}{|c|c|c|c|c|c|c|c|c|c|c|c|}
\hline \multirow[b]{3}{*}{ Gene } & \multirow[b]{3}{*}{ Start $^{1}$} & \multirow[b]{3}{*}{ Stop ${ }^{1}$} & \multirow[b]{3}{*}{ Length (bp) } & \multicolumn{8}{|c|}{ Flanking region ${ }^{2}\left(\mathrm{SNPs}^{3} /\right.$ total $\left.\mathrm{SNPs}^{4}\left(\mathrm{D}^{\prime}\right)^{5}\right)$} \\
\hline & & & & \multicolumn{4}{|c|}{ Without imputation } & \multicolumn{4}{|c|}{ With imputation } \\
\hline & & & & Affy5.0 & Affy6.0 & Illumina550K & Union & Affy5.0 & Affy6.0 & Illumina550K & Union \\
\hline HLA_A & $30,018,310$ & $30,021,632$ & 3,322 & $\begin{array}{c}200 \mathrm{~K} \\
(14 / 122(0.76))\end{array}$ & $\begin{array}{c}350 \mathrm{~K} \\
(16 / 299(0.69))\end{array}$ & $\begin{array}{c}150 \mathrm{~K} \\
(14 / 121(0.79))\end{array}$ & $\begin{array}{c}200 \mathrm{~K} \\
(16 / 329(0.74))\end{array}$ & $\begin{array}{c}150 \mathrm{~K} \\
(19 / 352(0.81))\end{array}$ & $\begin{array}{c}150 \mathrm{~K} \\
(17 / 364(0.80))\end{array}$ & $\begin{array}{c}200 \mathrm{~K} \\
(17 / 515(0.73))\end{array}$ & $\begin{array}{c}150 \mathrm{~K} \\
(16 / 366(0.80))\end{array}$ \\
\hline$H L A \_B$ & $31,429,630$ & $31,432,914$ & 3,284 & $\begin{array}{c}150 \mathrm{~K} \\
(20 / 100(0.61))\end{array}$ & $\begin{array}{c}100 \mathrm{~K} \\
(20 / 123(0.66))\end{array}$ & $\begin{array}{c}100 \mathrm{~K} \\
(18 / 131(0.59))\end{array}$ & $\begin{array}{c}100 \mathrm{~K} \\
(21 / 237(0.64))\end{array}$ & $\begin{array}{c}100 \mathrm{~K} \\
(24 / 282(0.67))\end{array}$ & $\begin{array}{c}40 \mathrm{~K} \\
(21 / 110(0.71))\end{array}$ & $\begin{array}{c}30 \mathrm{~K} \\
(22 / 102(0.71))\end{array}$ & $\begin{array}{c}40 \mathrm{~K} \\
(22 / 110(0.71))\end{array}$ \\
\hline HLA_C & $31,344,509$ & $31,347,834$ & 3,325 & $\begin{array}{c}150 \mathrm{~K} \\
(17 / 104(0.64))\end{array}$ & $\begin{array}{c}20 \mathrm{~K} \\
(14 / 25(0.83))\end{array}$ & $\begin{array}{c}10 \mathrm{~K} \\
(13 / 22(0.82))\end{array}$ & $\begin{array}{c}30 \mathrm{~K} \\
(17 / 95(0.79))\end{array}$ & $\begin{array}{c}30 \mathrm{~K} \\
(19 / 137(0.83))\end{array}$ & $20 \mathrm{~K}(18 / 88(0.85))$ & $\begin{array}{c}20 \mathrm{~K} \\
(16 / 88(0.85))\end{array}$ & $\begin{array}{c}20 \mathrm{~K} \\
(18 / 88(0.85))\end{array}$ \\
\hline HLA_DPB1 & $33,151,738$ & $33,162,954$ & 11,216 & $\begin{array}{c}100 \mathrm{~K} \\
(13 / 88(0.49))\end{array}$ & $\begin{array}{c}150 \mathrm{~K} \\
(15 / 154(0.47))\end{array}$ & $\begin{array}{c}100 \mathrm{~K} \\
(15 / 120(0.52))\end{array}$ & $\begin{array}{c}150 \mathrm{~K} \\
(18 / 280(0.47))\end{array}$ & $\begin{array}{c}20 \mathrm{~K} \\
(34 / 113(0.79))\end{array}$ & $\begin{array}{c}100 \mathrm{~K} \\
(33 / 352(0.54))\end{array}$ & $\begin{array}{c}150 \mathrm{~K} \\
(35 / 452(0.51))\end{array}$ & $\begin{array}{c}100 \mathrm{~K} \\
(35 / 355(0.54))\end{array}$ \\
\hline HLA_DQB1 & $32,735,635$ & $32,742,419$ & 6,784 & $\begin{array}{c}40 \mathrm{~K} \\
(12 / 15(0.61))\end{array}$ & $\begin{array}{c}40 \mathrm{~K} \\
(11 / 30(0.71))\end{array}$ & $\begin{array}{c}30 \mathrm{~K} \\
(12 / 13(0.72))\end{array}$ & $\begin{array}{c}30 \mathrm{~K} \\
(13 / 29(0.70))\end{array}$ & $\begin{array}{c}30 \mathrm{~K} \\
(16 / 52(0.69))\end{array}$ & $\begin{array}{c}30 \mathrm{~K} \\
(16 / 52(0.69))\end{array}$ & $\begin{array}{c}30 \mathrm{~K} \\
(15 / 52(0.69))\end{array}$ & $\begin{array}{c}30 \mathrm{~K} \\
(15 / 52(0.69))\end{array}$ \\
\hline HLA_DRB1 & $32,654,527$ & $32,665,559$ & 11,032 & $\begin{array}{c}200 \mathrm{~K} \\
(16 / 108(0.60))\end{array}$ & $\begin{array}{c}100 \mathrm{~K} \\
(16 / 27(0.73))\end{array}$ & $\begin{array}{c}150 \mathrm{~K} \\
(17 / 108(0.61))\end{array}$ & $\begin{array}{c}150 \mathrm{~K} \\
(21 / 204(0.61))\end{array}$ & $\begin{array}{c}150 \mathrm{~K} \\
(25 / 325(0.61))\end{array}$ & $\begin{array}{c}100 \mathrm{~K} \\
(22 / 91(0.76))\end{array}$ & $\begin{array}{c}200 \mathrm{~K} \\
(25 / 523(0.58))\end{array}$ & $\begin{array}{c}150 \mathrm{~K} \\
(24 / 326(0.62))\end{array}$ \\
\hline
\end{tabular}

${ }^{1}$ Based on NCBI build 36.3 .

${ }^{2}$ Flanking region for the most accurate prediction model.

${ }^{3}$ Number of SNPs within the region that were selected for the model (based on predictive power).

${ }^{4}$ Total number of SNPs in the MHC and HLA regions.

${ }^{5}$ Hedridge's multialleic D. 

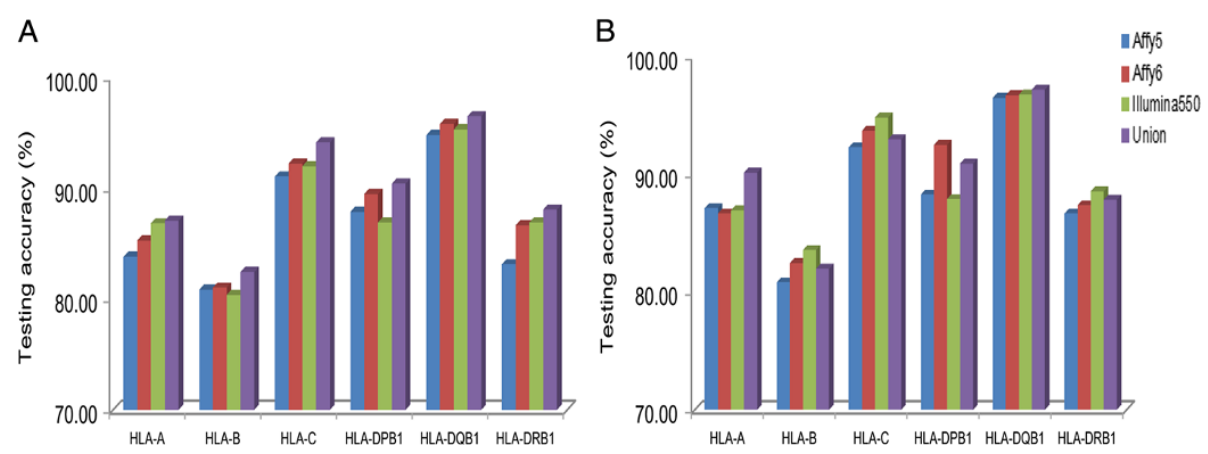

Figure 2 Testing accuracies for optimized models generated from each genotyping platform. Testing accuracies and call rates are shown for the six HLA genes (for $C T=0$ ). Values from each of the three genotyping arrays as well as from the union of the three arrays (colored bars) are shown both without (A) and with (B) imputed SNPs.

from different platforms (Additional file 7) and listed the alleles of the SNPs that predict HLA allele types (Additional file 8).

\section{Predictive accuracies with imputation}

We also performed comparisons between each pair of genotyping platforms to assess the concordance of SNPs with imputation. The Kappa coefficients were at least 0.9587 for these measurements, which were based on the genotypes originally observed and then imputed on Affy 5.0 and Illumina $550 \mathrm{~K}$ (Additional file 4). These results implied high imputation quality and comparable genotypes across the platforms.

In general, Union (average testing accuracy of $90.17 \%$ at $\mathrm{CT}=0$ ) generated more-accurate $H L A$-allele predictions than the three individual platforms (for $\mathrm{CT}=0$, average testing accuracies were $89.90 \%, 88.61 \%$, and $89.75 \%$ for Affy 6.0, Affy 5.0, and Illumina $550 \mathrm{~K}$, respectively; Figure 2B). As such, higher SNP densities may increase the accuracy of genotype imputation and, therefore, the accuracy of the final prediction. These results are consistent with Zhang et al.'s [7]. However, Illumina $550 \mathrm{~K}$ and Affy 6.0 sometimes yielded better accuracy than the union arrays in our results. One of the possible reasons might be due to "non-concordance" among these platforms. The kappa coefficients ranged from $95.87 \%$ to $97.62 \%$ (Additional file 4). It's likely that those with low concordance genotypes might decrease imputation accuracies.

In the comparisons between the three genotyping platforms with imputation, Affy 6.0 predictions were generally more accurate for $H L A$ alleles (up to $4.23 \%$ more accurate than Affy 5.0 , and $4.61 \%$ more accurate than Illumina $550 \mathrm{~K}$ at the $H L A-D P B 1$ locus; Figure 2B). Among these models $(C T=0)$, the $H L A-D Q B 1$ locus had the highest testing accuracy (96.75\%, from Illumina $550 \mathrm{~K}$ ), whereas the $H L A-B$ locus had the lowest (80.81\%, from Affy 5.0$)$. By applying a CT value of 0.9 to the maximum probability of all possible pairs of $H L A$ alleles, the highest accuracy was increased to $99.09 \%$ at the $H L A-C$ locus (with a call rate of $82.40 \%$, from Illumina $550 \mathrm{~K}$ ), and the lowest accuracy was increased to $91.58 \%$ at the $H L A-A$ locus (with a call rate of $41.65 \%$, from Affy 5.0). Besides $H L A-B$ from Affy 5.0, accuracy improvements based on this CT adjustment were most dramatic for the HLA-DRB1 gene, which rose from $86.67 \%$ to $95.90 \%$ when CT was changed from 0 to 0.9 . Affy 5.0 $(\mathrm{CT}=0)$, however, generated a prediction that was $0.45 \%$ more accurate than Affy 6.0 at the $H L A-A$. Besides $H L A$ DPB1 (with a more testing accuracy of $4.61 \%$ ), Illumina $550 \mathrm{~K}$ generated predictions that were $0.27 \%, 1.10 \%, 1.11 \%$, $0.05 \%$ and $1.19 \%$ more accurate than Affy 6.0 at the HLA$A,-B,-C,-D Q B 1$ and $-D R B 1$ loci, respectively (Figure 2). These findings are consistent with Zhang et al.'s [7], which showed that prediction improvements from the Affy 5.0 and Illumina $550 \mathrm{~K}$ array data may be attributed to SNPs that are exclusively represented on these platforms. Details concerning testing accuracies and prediction call rates across different platforms and different CTs are listed in Additional file 5 .

For each $H L A$ locus, we also assessed the sufficient flanking region with imputation. One of the shortest flanking regions was identified by Affy 5.0 at the $H L A$ $D P B 1$ locus $( \pm 20 \mathrm{~kb})$ (Table 1$)$. This region covered 113 SNPs, of which 34 were selected for the HLA-DPB1prediction model (testing accuracy of $88.28 \%$ at $\mathrm{CT}=0$ ). The other shortest flanking regions were identified by Affy 6.0 , Illumina $550 \mathrm{~K}$ and Union at the $H L A-C$ locus $( \pm 20 \mathrm{~kb})$. The longest sufficient flanking region was $\pm 200 \mathrm{~kb}$ at $H L A-A$ from Illumina $550 \mathrm{~K}$; Table 1 ). Within this region there were 515 SNPs, of which 17 were used in the $H L A$-A-prediction model (testing accuracy of $86.93 \%$ at $\mathrm{CT}=0$ ).

For each $H L A$ gene, the amount of overlap among genotypes used by the different prediction models was at most $60.08 \%$ (Additional file 6). Imputation, therefore, seemed to reduce discrepancies among the different platforms. 


\section{Differences in predictive accuracies with and without imputation}

We performed comparisons between each pair of genotyping platform to assess overall concordance between SNPs with and without imputation. The lowest kappa coefficient was 0.9922, which was derived from Affy 5.0 genotypes (Additional file 4). These results demonstrated high imputation quality, even though nearly 10,000 SNPs within this $6-\mathrm{Mb}$ region were imputed from the Affy 5.0 chip.

Comparisons of testing accuracies among prediction models with and without imputation across different platforms revealed that prediction models built with imputed SNPs were more accurate than those built without imputed SNPs (average accuracies of $89.61 \%$ and $88.30 \%$, respectively, for $\mathrm{CT}=0$; Figure 2). Prediction improvements associated with imputation of the HLA-DRB1 alleles on Affy 5.0 were more dramatic than those seen with other HLA genes across the three platforms. Imputation at this locus elevated prediction accuracy from $83.14 \%$ to $86.67 \%$. These results essentially agree with Zhang et al.'s [7], who suggested that more SNPs generally increased $H L A$ allele-prediction accuracy.

For $\mathrm{CT}=0$, the Union platform with imputation had the highest testing accuracy $(97.18 \%)$ for $H L A-D Q B 1$ alleles, whereas Illumina $550 \mathrm{~K}$ without imputation had the lowest testing accuracy (80.37\%) for $H L A-B$ alleles. By applying a CT of 0.9 to the maximum probability of all possible pairs of $H L A$ alleles, the highest accuracy was elevated to $99.09 \%$ at the $H L A-C$ locus with Illumina $550 \mathrm{~K}$ (with imputation and a call rate of $82.40 \%$ ). This CT adjustment increased the lowest accuracy to $87.67 \%$ at the $H L A-B$ locus with Affy 5.0 (without imputation and with a call rate of 64.94\%; Additional file 5).

Comparing testing-prediction accuracies with and without imputation among the different platforms, indicated genotype variation generally decreased when imputed SNPs were used to build the prediction model of $H L A$ genes. For each $H L A$ gene among the different platforms, the percentage overlap among genotypes that were selected to build the models increased $25.02 \%$ on average with imputation (Additional file 6). These results may have stemmed from using a consistent set of HapMap SNPs, which could have minimized discrepancies among different genotyping platforms.

\section{Discussion}

Because classic technologies for the direct typing of $H L A$ alleles are economically infeasible, we instead applied a method developed by Li et al. [13], which identifies specific $H L A$ alleles based on their corresponding unphased genotypes. We used this method to build prediction model of $H L A$ genes for $H L A$ class I (HLA-A, HLA-B, and $H L A-C)$ and class II (HLA-DRB1, HLA-DQB1, and $H L A-D P B 1)$ genes. We compared allele frequency distributions of $H L A$ genes between the Han Chinese from Taiwan and the Caucasian population (from the HapMap Project) Allele frequencies are different between our Han Chinese and the Caucasian samples $\mathrm{p}<0.0001$ with chi-square and Fisher's exact tests. That is because some of the alleles are the same but just at different frequencies. The most polymorphic $H L A$ locus was $H L A-B$. Within the $H L A-B$ region, we identified more alleles in the Han Chinese population (44 alleles) than in the Caucasian HapMap population (32 alleles). This allele frequency difference supports the use of the method of Li et al. [13], as well as the use of a Taiwan-based data set to build ethnicspecific prediction model of HLA genes for a Han Chinese population. We have built a number of models for predicting class I and class II HLA genes at a four-digit resolution and have examined critical parameters associated with these models (e.g., the sufficient flanking region, platform accuracy, and the effect of imputation). We found that our models accurately predict these alleles within a Han Chinese population. Results from this study have direct implications for detailed analyses of $H L A$-related disease associations.

$H L A$ genotypes were observed from our previous study [15]. Briefly, six classic $H L A$ loci $(H L A-A,-B,-C$, $D Q B 1,-D R B 1$, and $-D P B 1$ ) to a 4-digit resolution were analyzed in the present study. We did not include $D Q A 1$ or $D R B 3,4$, and 5 in this study, partly because of the unavailability of genotyping kits and partly because that their LD with corresponding $D Q B 1$ or $D R B 1$ alleles would be too tight to be delineated. In some of our previous studies, we reported that $H L A-B^{*} 15: 02$ was highly associated with Carbamazepine-induced Stevens-Johnson syndrome and toxic epidermal necrolysis [38-41].

$H L A-B$ had the highest overlapping density between our Han Chinese and the Caucasian population ( $\mathrm{Li}$ et al. [13]), the number of predictive SNPs was 69 for the former and 86 for the latter. Among the 155 predictive SNPs, the number of the overlapping SNPs was 11. $H L A-D R B 1$ had the lowest overlapping density (4 overlapping SNPs), in which, our Han Chinese required 51 predictive SNPs and the Caucasian population required 41 predictive SNPs.

In an attempt to differentiate between the effects of the Li et al.'s algorithm and the Han Chinese panel, we examined the HLA predictive accuracies between the two algorithms proposed by $\mathrm{Li}$ et al. (2011) and Leslie et al. (2008) and found that the paper reported by $\mathrm{Li}$ et al. (2011) has shown that the $H L A$ predictive accuracies for the two algorithms were in general comparable using the British 1958 birth cohort data. We also applied the Li et al.'s method to HapMap CEU/CEPH data, we compared the accuracies derived from Li et al.'s method with those by Leslie et al.'s We built $H L A-B$ and $H L A-D Q B 1$ models 
(the worst and the best performance models, respectively) using leave-one-out CV adapted by Leslie et al. With a CT of 0.9 , accuracy of $H L A-B$ was $100 \%$ from Li et al. and 95\% from Leslie et al. For $H L A-D Q B 1$, accuracy was 99\% from $\mathrm{Li}$ et al. and 99\% from Leslie et al. Therefore, we concluded that the prediction models were generally comparable for the two methods.

In this study, the SNP genotype imputation is used as material preparation for the $H L A$ allele prediction. The imputation of the SNP genotypes refers to increasing the SNP density by adding SNPs that originally are not incorporated in the array chips but in the HapMap project data (Chinese Han Beijing (CHB) + Japanese Tokyo (JPT) in our case). The HapMap project data mainly includes Chinese Han Beijing ( $\mathrm{CHB}$ ) belonging to northern Chinese Han subgroup. Imputation was carried out to increase the diversity of alleles covered in our training data and also help to boost the accuracy for predicting $H L A$ genotypes. The missing rate for our HLA data was very low $(0.23 \%)$, therefore, no imputation was performed for the $H L A$ genotype data. Here we determined whether a denser collection of SNPs would generate more-accurate HLA allele predictions. The $\mathrm{MaCH}$ software, which we used to impute our chip data sets and thus build greater SNP density, uses data from the HapMap Project and/or the 1000 Genomes Project (http://www.1000genomes.org) as references. We found that prediction model of $H L A$ genes that were built with imputation typically provided greater prediction accuracy, which underscores the positive effect of using a higher density of SNPs. Therefore, it is possible to devise a new customized SNP array that includes all of the selected SNPs consisting those of genotyped or imputed SNPs for the $H L A$-prediction model to increase the prediction accuracy. Additionally, we used our genotype data from Affy 6.0 (the densest chip data) to build $H L A-B$ and $H L A-D Q B 1$ models (the worst and the best performance models, respectively) using a 2-fold CV. The imputation was carried out separately for training and testing data set. We then compared the results with those obtained by imputation simultaneously for the two data sets. The testing accuracy of $H L A-B$ for separated imputation was $90 \%$ and it was $89 \%$ for simultaneous imputation. For $H L A-D Q B 1$, the testing accuracies for separated imputation and for simultaneous imputation were both $96 \%$.

By increasing CT to 0.5 or 0.9 , levels of prediction accuracy approached $100 \%$ in our ethnic-specific prediction model of HLA genes for Han Chinese populations (Additional file 5). We analyzed the effect, therefore, of different reference data sets beyond our Affy 6.0 genotype data. When using the 1000 Genomes Project as the reference at $\mathrm{CT}=0$, we found that testing accuracy concerning the $H L A-C$ gene decreased by $3.02 \%$ (down to $90.17 \%)$. Similar trends were observed at the other five $H L A$ loci. Rare SNP variants within data from the 1000
Genomes Project may have increased the number of mismatched genotypes under imputation.

To generate more-accurate prediction models, we varied sample sizes for both the training and testing data sets. We used our genotype data from Affy 6.0 to build prediction model of HLA genes using 2-, 4-, and 10-fold CV. With CT $=0$ (i.e., a call-rate of $100 \%$ ) we obtained a consistent sample size for the different CVs. To eliminate the effect of sample size, therefore, we compared effects of $\mathrm{CV}$ at $\mathrm{CT}=0$. Additional file 9 shows the estimates for the testing accuracy that was obtained from validation analyses of prediction models built using 2-, 4-, and 10-fold CV (for $\mathrm{CT}=0$ ). The $H L A-D Q B 1$ locus with 10 -fold $\mathrm{CV}$ had the best testing accuracy (95.55\%), whereas the $H L A-B$ locus with 2-fold $\mathrm{CV}$ had the lowest testing accuracy (76.64\%). At the 2-fold $\mathrm{CV}$, testing accuracies ranged from $76.64 \%$ (HLA-B) to $94.39 \%$ (HLA-DQB1). By increasing the CV to 10-fold, testing-accuracy levels approached 95.55\% (HLA$D Q B 1)$. For $H L A-A$, this improvement was most dramatic, as prediction models trained on the 2 -fold $\mathrm{CV}$ had an accuracy of $80.84 \%$, whereas training on the 10 -fold CV resulted in an $85.29 \%$ accuracy. Similar trends were observed for the other five $H L A$ loci. These trends may reflect the large size of the sample, which would include a sufficient number of $H L A$ alleles in the training data set, thereby increasing predictive accuracy. Although the level of CV influenced the testing accuracy, these changes were minimal. We conclude, therefore, that our ethnic-specific prediction model of HLA genes is not affected by different CVs.

For $H L A-B$ alleles, testing accuracies were generally not as high as others. One of the possible reasons was that there were large number of $H L A-B$ allele with four digit and most of them with very low frequency. Hence, our training data could not sufficiently capture the variability. We also compared testing accuracies among predictive models with and without imputation across different platforms, the results revealed that predictive models built with imputed SNPs were more accurate than those without (average accuracies of $89.61 \%$ and $88.30 \%$, respectively, for $\mathrm{CT}=0$; Figure 2). Therefore, proxy SNPs might improve testing accuracies. To generate more-useful prediction model of $H L A$ genes, therefore, one should take into account that the distribution of these rare $H L A$ alleles varies between the training and testing data sets. As such, applying the current $H L A$-predictive method to a large population that contains relatively rare $H L A$ alleles in the training data set might improve the performance of ethnic-specific prediction model of HLA genes.

The common $H L A$ typing experiment is not restricted to a single $H L A$ allele but to a group of known $H L A$ alleles having the same pattern within the common allele. Therefore, it is hard to determine rare alleles. However, we feel the prediction model is a useful tool for prescreening disease association with common $H L A$ alleles 
using chip data from GWAS. In the case of any significant association by the model predicted common alleles, it is understood that further $H L A$ testing would be necessary. The $H L A$ allele assignment was according to the most common alleles (allele frequency $>0.01$ ) found in Taiwanese populations and southern Chinese populations as determined in the population studies of $13^{\text {th }}$ international histocompatibility workshop.

As mentioned above, we focused on prediction model of HLA genes that were generated using 214 Han Chinese samples from Taiwan, each of which were genotyped using the Affy 5.0, Affy 6.0, and Illumina $550 \mathrm{~K}$ chips. To assess the effect of the sample size for predicting, we analyzed prediction models generated using 437 Han Chinese samples (which included the original 214 samples) that were genotyped using the Illumina $550 \mathrm{~K}$ chip. Prediction model of HLA genes built from 437 samples (average testing accuracy was 90.36\%) performed better than that from the 214-sample (average testing accuracy was $86.84 \%$ ) across all $H L A$ loci. As such, larger sample sizes seem to increase $H L A$-predictive accuracy. The sample size effects was confirmed by the previous finding from Jia et al. [11]. In our previous study [17], the profiles of haplotype blocks for the Caucasian and Taiwanese populations were apparently different in LD structure. Our HLA typing experiment assignment was according to the most common alleles (allele frequency > 0.01), but the Caucasian HLA data (Li et al. [13]) did not filter rare alleles. Inaccurate predictions might have resulted from rare HLA alleles, which were defined as those appeared less than three times in the training data set [13]. These rare $H L A$ alleles might affect the first procedure of $H L A$-predictive methodology [13]. Hence, sample size, LD and configuration of allelic repertoire might be all possible reasons for the difference in the size of optimal flanking region for our Han Chinese and the Caucasian data.

We offer a web-based service on the HLA predictive models using Li's algorithm to help other researchers to predict the $H L A$ allele types and the corresponding probabilities of their subjects if the researchers provide the SNPs information files on the $H L A$ region to us. The link is at the following (http://www.csjfann.ibms.sinica. edu.tw/eag/programlist/program_list.html).

\section{Conclusions}

After a decade of research, many HLA genes are known to have specific immunological functions. Experimental methodologies that link SNPs to HLA alleles offer considerable savings (both in time and expense) over direct $H L A$-typing technologies and make large-scale investigations into $H L A$ variation feasible. Although $H L A$ allelic distributions differ among human populations, the majority of existing prediction model of $H L A$ genes is based on Caucasian samples. By genotyping a large number of Han Chinese samples, we have uncovered many HLA alleles that are unique to this population and have built ethnic-specific prediction model of $H L A$ genes. Our training data set covers many uncommon and ethnicspecific alleles within $H L A$ loci, substantially raising predictive accuracies concerning the testing of data sets.

Specific methodological parameters investigated in this study (e.g., sample size, SNP density, and imputation) each were factors in generating prediction model of $H L A$ genes that were specific for a Han Chinese population. We achieved good predictive accuracy for the $H L A-A,-B,-C$, $-D R B 1,-D Q B 1$, and $-D P B 1$ genes within our Han Chinese samples. Using SNP data from the Affymetrix GenomeWide Human SNP Array 5.0, the Affymetrix GenomeWide Human SNP Array 6.0, the Illumina HumanHap550 BeadChip, or a union of these three technological platforms, we generated efficient prediction model of $H L A$ genes for determining $H L A$ alleles within a Han Chinese population. Our novel predictive tools may help identify genetic risk factors for immune-related diseases. Furthermore, these findings will enable researchers to investigate $H L A$ allelic variations among a broader range of human populations.

\section{Additional files}

\begin{abstract}
Additional file 1: Overlapping SNPs between the HapMap Project and three genotyping platforms within the extended MHC region. Additional file 2: List of the $H L A$ alleles and allele frequencies in our Han Chinese data $(n=214)$.

Additional file 3: Testing accuracies associated with different flanking-region sizes. For each of the six HLA genes (colored lines), testing accuracies for various flanking-region sizes are shown. Data using the Affy 5.0 (A), Illumina $550 \mathrm{~K}(\mathrm{~B})$, and Union (C) chip data sets are shown. [Union refers to a union of data from the three platforms (Affy 5.0, Affy 6.0, and Illumina $550 \mathrm{~K})$ ].
\end{abstract}

Additional file 4: Kappa coefficients of observed or imputed SNP genotypes within the MHC region.

Additional file 5: Testing accuracies and call rates from optimized models for each platform at different CT settings. For each of the six HLA genes, testing accuracies and call rates are shown for the four genotyping arrays (colored bars). Data from $C T=0$ (blue), $C T=0.5$ (red), and $C T=0.9$ (gray) are shown. Panels represent data without imputed SNPs (A) and with imputed SNPs (B).

Additional file 6: The percentage of overlapping selected genotypes between the different genotyping platforms. For each of the six HLA genes (colored bars), the percentage of overlapping SNPS from different pairs of arrays is shown. Data without imputed SNPs (A) and with imputed SNPS (B) are shown.

Additional file 7: List of the selected SNPs in the final HLA prediction models.

Additional file 8: List of the SNP alleles that predict $H L A$ allele types.

Additional file 9: Testing accuracies with different CV settings. For each of the six HLA genes, testing accuracies associated with 10-fold CV (blue), 4-fold CV (red), and 2-fold CV (green) are shown (for CT =0).

Competing interests

The authors declare that they have no competing interests. 


\section{Authors' contributions}

ARH and SWC contribute equally. ARH, SWC, CLH, CCC and CSJF contributed to the statistical analysis. ARH and CSJF drafted the manuscript. PLC, CCC and SWC contributed to help to draft the manuscript with ARH. PLC, CCC, WSY, JYW, YTC and TCC contributed to collecting the data. All authors read and approved the final manuscript.

\section{Acknowledgements}

We thank the National Center for Genome Medicine at Academia Sinica, Taiwan, for technical/bioinformatics support. This Center was supported by grants from the National Core Facility Program for Biotechnology of National Science council, Taiwan. We are also grateful to National Science Council and Institute of Biomedical Sciences, Academia Sinica of Taiwan and National Taiwan University Hospital for their funding (NSC101-2314-B-001-001, NSC 100-2325-B-002-015, and NSC100-2314-B-002-118-MY3).

\section{Author details}

IInstitute of Biomedical Sciences, Academia Sinica, Nankang, Taipei, Taiwan. ${ }^{2}$ Clinical Informatics and Medical Statistics Research Center, Chang Gung University College of Medicine, Taoyuan, Taiwan. ${ }^{3}$ Department of Medical Genetics, National Taiwan University Hospital, Taipei, Taiwan. ${ }^{4}$ Department of Internal Medicine, National Taiwan University Hospital, Taipei, Taiwan. ${ }^{5}$ Graduate Institute of Clinical Medicine, College of Medicine, National Taiwan University, Taipei, Taiwan. ${ }^{6}$ Department of Internal Medicine, College of Medicine, National Taiwan University, Taipei, Taiwan. ${ }^{7}$ National Genotyping Center, Academia Sinica, Taipei, Taiwan. ${ }^{8}$ Graduate Institute of Chinese Medical Science, China Medical University, Taichung, Taiwan. ${ }^{9}$ Department of Pediatrics, Duke University Medical Center, Durham, North Carolina, USA ${ }^{10}$ Immunogenetics laboratory, Medical Research Department, Mackay Memorial Hospital, Taipei, Taiwan. ${ }^{11}$ Research Center for Developmental Biology and Regenerative Medicine, National Taiwan University, Taipei, Taiwan. ${ }^{12}$ Graduate Institute of Medical Genomics and Proteomics, National Taiwan University College of Medicine, Taipei, Taiwan. ${ }^{13}$ Graduate Institute of Biostatistics, China Medical University, Taichung, Taiwan.

Received: 15 August 2013 Accepted: 16 January 2014 Published: 29 January 2014

\section{References}

1. Claas FH, Duquesnoy RJ: The polymorphic alloimmune response in clinical transplantation. Curr Opin Immunol 2008, 20(5):566-567.

2. Lee SJ, Klein J, Haagenson M, Baxter-Lowe LA, Confer DL, Eapen M, Fernandez-Vina M, Flomenberg N, Horowitz M, Hurley CK, et al: Highresolution donor-recipient HLA matching contributes to the success of unrelated donor marrow transplantation. Blood 2007, 110(13):4576-4583.

3. Asano K, Matsushita T, Umeno J, Hosono N, Takahashi A, Kawaquchi T, Matsumoto T, Matsui T, Kakuta Y, Kinouchi Y, et al: A genome-wide association study identifies three new susceptibility loci for ulcerative colitis in the Japanese population. Nat Genet 2009, 41(12):1325-1329.

4. Hirschfield GM, Liu X, Xu C, Lu Y, Xie G, Gu X, Walker EJ, Jing K, Juran BD, Mason AL, et al: Primary biliary cirrhosis associated with HLA, IL12A, and IL12RB2 variants. N Engl J Med 2009, 360(24):2544-2555.

5. Evseeva I, Nicodemus KK, Bonilla C, Tonks S, Bodmer WF: Linkage disequilibrium and age of HLA region SNPs in relation to classic HLA gene alleles within Europe. Eur J Hum Genet 2010, 18(8):924-932.

6. Barreiro $L B$, Quintana-Murci $L$ : From evolutionary genetics to human immunology: how selection shapes host defence genes. Nat Rev Genet 2010, 11(1):17-30.

7. Zhang XC, Li SS, Wang H, Hansen JA, Zhao LP: Empirical evaluations of analytical issues arising from predicting HLA alleles using multiple SNPs. BMC Genet 2011, 12:39.

8. Tang M, Lautenberger JA, Gao X, Sezgin E, Hendrickson SL, Troyer JL, David VA, Guan L, McIntosh CE, Guo X, et al: The principal genetic determinants for nasopharyngeal carcinoma in China involve the HLA class I antigen recognition groove. PLOS Genet 2012, 8(11):e1003103.

9. Lin M, Chu CC, Chang SL, Lee HL, Loo JH, Akaza T, Juji T, Ohashi J. Tokunaga K: The origin of Minnan and Hakka, the so-called "Taiwanese", inferred by HLA study. Tissue Antigens 2001, 57(3):192-199.

10. Wang LM, Kimura A, Satoh M, Mineshita S: HLA linked with leprosy in southern China: HLA-linked resistance alleles to leprosy. Int J Lepr Other Mycobact Dis 1999, 67(4):403-408.
11. Jia X, Han B, Onengut-Gumuscu S, Chen WM, Concannon PJ, Rich SS, Raychaudhuri S, de Bakker PI: Imputing amino acid polymorphisms in human leukocyte antigens. PLOS One 2013, 8(6):e64683.

12. Xie M, Li J, Jiang T: Accurate HLA type inference using a weighted similarity graph. BMC Bioinforma 2010, 11(Suppl 11):S10.

13. Li SS, Wang H, Smith A, Zhang B, Zhang XC, Schoch G, Geraghty D, Hansen JA, Zhao LP: Predicting multiallelic genes using unphased and flanking single nucleotide polymorphisms. Genet Epidemiol 2011, 35(2):85-92.

14. Leslie $S$, Donnelly P, McVean G: A statistical method for predicting classical HLA alleles from SNP data. Am J Hum Genet 2008, 82(1):48-56.

15. Fasil TA, Hailu E, Finan C, Aseffa A, Davey G, Newport MJ, Rotimi CN, Adeyemo A: Prediction of HLA class II alleles using SNPs in an African population. PLoS One 2012, 7(6):pg e40206.

16. Pan WH, Fann CS, Wu JY, Hung YT, Ho MS, Tai TH, Chen YJ, Liao CJ, Yang ML, Cheng AT, et al: Han Chinese cell and genome bank in Taiwan: purpose, design and ethical considerations. Hum Hered 2006, 61(1):27-30.

17. Yang HC, Lin $\mathrm{CH}$, Hsu CL, Hung SI, Wu JY, Pan WH, Chen YT, Fann CS: A comparison of major histocompatibility complex SNPs in Han Chinese residing in Taiwan and Caucasians. J Biomed Sci 2006, 13(4):489-498.

18. Chen PL, Fann CS, Chu CC, Chang CC, Chang SW, Hsieh HY, Lin M, Yang WS, Chang TC: Comprehensive genotyping in two homogeneous Graves' disease samples reveals major and novel HLA association alleles. PLOS One 2011, 6(1):e16635.

19. Koehler $A B$, Murphree ES: A comparison of the akaike and schwarz criteria for selecting model order. App/ Stat-J Roy St C 1988, 37(2):187-195.

20. Fernandez Vina MA, Hollenbach JA, Lyke KE, Sztein MB, Maiers M, Klitz W Cano P, Mack S, Single R, Brautbar C, et al: Tracking human migrations by the analysis of the distribution of HLA alleles, lineages and haplotypes in closed and open populations. Phil Trans R Soc Lond B Biol Sci 2012, 367(1590):820-829.

21. International HapMap C, Frazer KA, Ballinger DG, Cox DR, Hinds DA, Stuve LL, Gibbs RA, Belmont JW, Boudreau A, Hardenbol P, et al: A second generation human haplotype map of over 3.1 million SNPs. Nature 2007, 449(7164):851-861

22. Matsuzaki H, Dong S, Loi H, Di X, Liu G, Hubbell E, Law J, Berntsen T, Chadha M, Hui H, et al: Genotyping over 100,000 SNPs on a pair of oligonucleotide arrays. Nat Methods 2004, 1(2):109-111.

23. Kennedy GC, Matsuzaki H, Dong S, Liu WM, Huang J, Liu G, Su X, Cao M, Chen W, Zhang J, et al: Large-scale genotyping of complex DNA. Nat Biotechnol 2003, 21(10):1233-1237.

24. Steemers FJ, Chang W, Lee G, Barker DL, Shen R, Gunderson KL: Whole-genome genotyping with the single-base extension assay. Nat Methods 2006, 3(1):31-33

25. Steemers FJ, Gunderson KL: Whole genome genotyping technologies on the BeadArray platform. Biotechnol J 2007, 2(1):41-49.

26. Cohen J: A coefficient of agreement for nominal scales. Educ Psychol Meas 1960, 20(1):37-46.

27. Almeria S, Vidal D, Ferrer D, Pabon M, Fernandez-de-Mera MI, Ruiz-Fons F, Alzaga V, Marco I, Calvete C, Lavin S, et al: Seroprevalence of Neospora caninum in non-carnivorous wildlife from Spain. Vet Parasitol 2007 143(1):21-28.

28. Howie B, Fuchsberger C, Stephens M, Marchini J, Abecasis GR: Fast and accurate genotype imputation in genome-wide association studies through pre-phasing. Nat Genet 2012, 44:955-959.

29. Howie B, Marchini J, Stephens M: Genotype imputation with thousands of genomes. G3 2011, 1(6):457-470.

30. Mijatovic V, lacobucci I, Sazzini M, Xumerle L, Mori A, Pignatti PF, Martinelli G, Malerba G: Imputation reliability on DNA biallelic markers for drug metabolism studies. BMC Bioinforma 2012, 13(Suppl 14):S7.

31. Li Y, Willer CJ, Ding J, Scheet $P$, Abecasis GR: MaCH: using sequence and genotype data to estimate haplotypes and unobserved genotypes. Genet Epidemiol 2010, 34(8):816-834

32. International HapMap C: Integrating ethics and science in the International HapMap Project. Nat Rev Genet 2004, 5(6):467-475.

33. Southam L, Panoutsopoulou K, Rayner NW, Chapman K, Durrant C, Ferreira T, Arden N, Carr A, Deloukas P, Doherty M, et al: The effect of genome-wide association scan quality control on imputation outcome for common variants. Eur J Hum Genet 2011, 19(5):610-614.

34. Marchini J, Howie B: Genotype imputation for genome-wide association studies. Nat Rev Genet 2010, 11(7):499-511. 
35. Lin P, Hartz SM, Zhang Z, Saccone SF, Wang J, Tischfield JA, Edenberg HJ, Kramer JR AMG, Bierut $L$, et al: A new statistic to evaluate imputation reliability. PLoS One 2010, 5(3):e9697.

36. Ng AY: Preventing "Overfitting" of Cross-Validation Data. In Proceedings of the Fourteenth International Conference on Machine Learning; 1997:245-253. http://ai.stanford.edu/ ang/papers/cv-final.pdf.

37. Sham PC, Curtis D: Monte Carlo tests for associations between disease and alleles at highly polymorphic loci. Ann Hum Genet 1995, 59(Pt 1):97-105.

38. Yang CW, Hung SI, Juo CG, Lin YP, Fang WH, Lu IH, Chen ST, Chen YT: HLA-B*1502-bound peptides: implications for the pathogenesis of carbamazepine-induced Stevens-Johnson syndrome. J Allergy Clin Immunol 2007, 120(4):870-877.

39. Wei $C Y$, Chung WH, Huang HW, Chen YT, Hung SI: Direct interaction between HLA-B and carbamazepine activates T cells in patients with Stevens-Johnson syndrome. J Allergy Clin Immunol 2012, 129(6):1562-1569. e1565.

40. Chung WH, Hung SI, Hong HS, Hsih MS, Yang LC, Ho HC, Wu JY, Chen YT: Medical genetics: a marker for Stevens-Johnson syndrome. Nature 2004, 428(6982):486.

41. Chen P, Lin JJ, Lu CS, Ong CT, Hsieh PF, Yang CC, Tai CT, Wu SL, Lu CH, Hsu YC, et al: Carbamazepine-induced toxic effects and HLA-B*1502 screening in Taiwan. N Engl J Med 2011, 364(12):1126-1133.

doi:10.1186/1471-2164-15-81

Cite this article as: Hsieh et al: Predicting HLA genotypes using unphased and flanking single-nucleotide polymorphisms in Han Chinese population. BMC Genomics 2014 15:81.

\section{Submit your next manuscript to BioMed Central and take full advantage of:}

- Convenient online submission

- Thorough peer review

- No space constraints or color figure charges

- Immediate publication on acceptance

- Inclusion in PubMed, CAS, Scopus and Google Scholar

- Research which is freely available for redistribution 